\title{
Enders, Livia; Winter, Stephanie
}

\section{"Das hatte ich mir anders vorgestellt". Studierende und Dozierende im} Spannungsfeld zwischen Theorie und Praxis. Die Hochschullernwerkstatt als Forschungsraum

Kramer, Kathrin [Hrsg.]; Rumpf, Dietlinde [Hrsg.]; Schöps, Miriam [Hrsg.]; Winter, Stephanie [Hrsg.]: Hochschullernwerkstätten - Elemente von Hochschulentwicklung? Ein Rückblick auf 15 Jahre Hochschullernwerkstatt in Halle und andernorts. Bad Heilbrunn : Verlag Julius Klinkhardt 2020, S. 129-144. - (Lernen und Studieren in Lernwerkstätten)

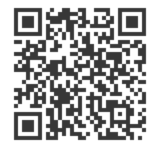

Quellenangabe/ Reference:

Enders, Livia; Winter, Stephanie: "Das hatte ich mir anders vorgestellt". Studierende und Dozierende im Spannungsfeld zwischen Theorie und Praxis. Die Hochschullernwerkstatt als Forschungsraum - In: Kramer, Kathrin [Hrsg.]; Rumpf, Dietlinde [Hrsg.]; Schöps, Miriam [Hrsg.]; Winter, Stephanie [Hrsg.]: Hochschullernwerkstätten - Elemente von Hochschulentwicklung? Ein Rückblick auf 15 Jahre Hochschullernwerkstatt in Halle und andernorts. Bad Heilbrunn : Verlag Julius Klinkhardt 2020, S. 129-144 - URN: urn:nbn:de:0111-pedocs-212073 - DOI: 10.25656/01:21207

https://nbn-resolving.org/urn:nbn:de:0111-pedocs-212073

https://doi.org/10.25656/01:21207

in Kooperation mit / in cooperation with:

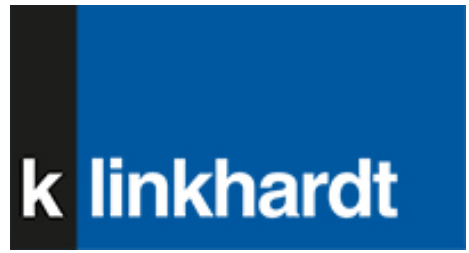

http://www.klinkhardt.de

\section{Nutzungsbedingungen}

Dieses Dokument steht unter folgender Creative Commons-Lizenz: http://creativecommons.org/licenses/by-nc-sa/4.0/deed.de - Sie dürfen das Werk bzw. den Inhalt unter folgenden Bedingungen vervielfältigen, verbreiten und öffentlich zugänglich machen sowie Abwandlungen und Bearbeitungen des Werkes bzw. Inhaltes anfertigen: Sie müssen den Namen des Autors/Rechteinhabers in der von ihm festgelegten Weise nennen. Dieses Werk bzw. der Inhalt darf nicht für kommerzielle Žwecke verwendet werden. Die neu entstandenen Werke bzw. Inhalte dürfen nur unter Verwendung von Lizenzbedingungen weitergegeben werden, die mit denen dieses Lizenzvertrages identisch oder vergleichbar sind.

Mit der Verwendung dieses Dokuments erkennen Sie die Nutzungsbedingungen an.

\section{Terms of use}

This document is published under following Creative Commons-License: http://creativecommons.org/licenses/by-nc-sa/4.0/deed.en - You may copy, distribute and transmit, adapt or exhibit the work in the public and alter, transform or change this work as long as you attribute the work in the manner specified by the author or licensor. You are not allowed to make commercial use of the work. If you alter, transform, or change this work in any way, you may distribute the resulting work only under this or a comparable license.

By using this particular document, you accept the above-stated conditions of use.

\section{Kontakt / Contact:}

pedocs

DIPF | Leibniz-Institut für Bildungsforschung und Bildungsinformation

Informationszentrum (IZ) Bildung

E-Mail: pedocs@dipf.de

Internet: www.pedocs.de

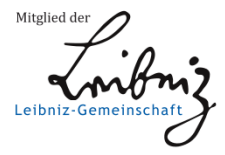




\section{Lernen und Studieren in Lernwerkstätten}

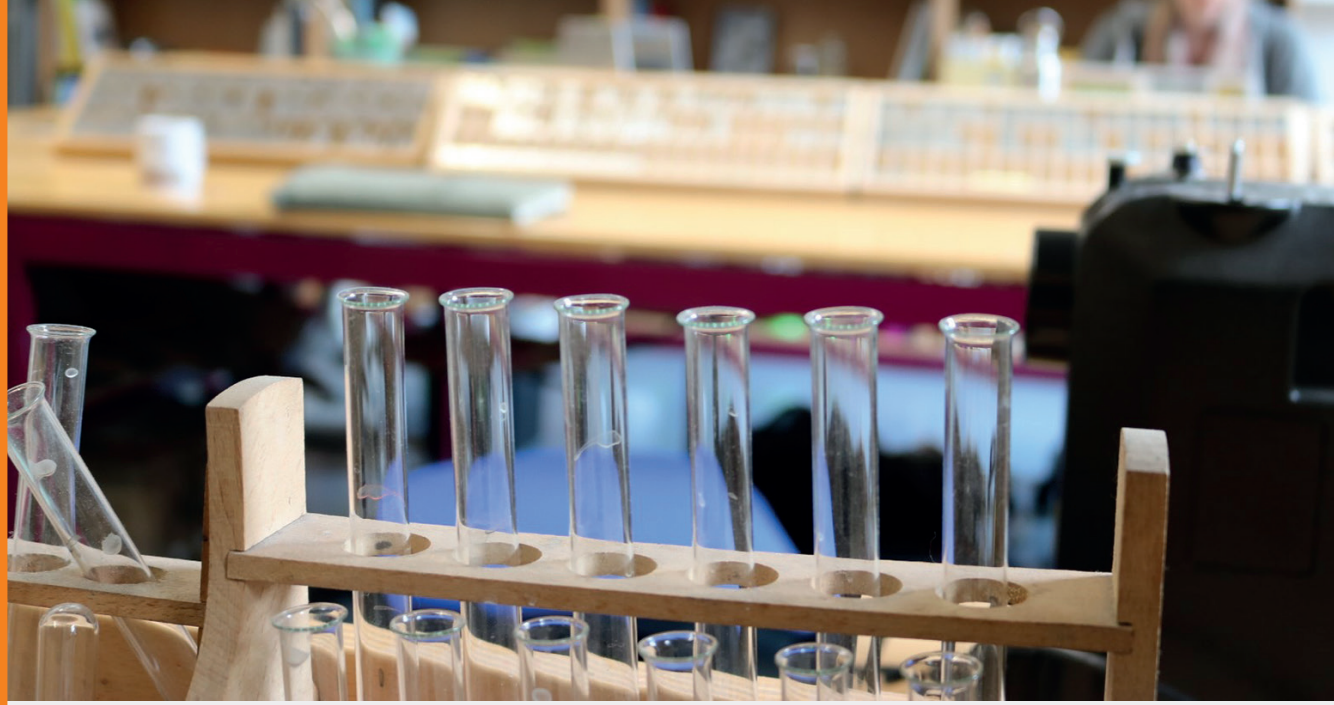

Kathrin Kramer / Dietlinde Rumpf / Miriam Schöps / Stephanie Winter (Hrsg.)

Hochschullernwerkstätten - Elemente von Hochschulentwicklung?

Ein Rückblick auf 15 Jahre Hochschullernwerkstatt in Halle und andernorts 


\section{Kramer / Rumpf / Schöps / Winter Hochschullernwerkstätten - Elemente von Hochschulentwicklung?}




\section{Lernen und Studieren in Lernwerkstätten}

Impulse für Theorie und Praxis

Herausgegeben von

Eva-Kristina Franz, Johannes Gunzenreiner, Barbara Müller-Naendrup, Hartmut Wedekind und Markus Peschel 


\section{Kathrin Kramer \\ Dietlinde Rumpf \\ Miriam Schöps \\ Stephanie Winter \\ (Hrsg.)}

\section{Hochschullernwerkstätten - Elemente von Hochschulentwicklung?}

Ein Rückblick auf 15 Jahre Hochschullernwerkstatt in Halle und andernorts 
Der vorliegende Band ist anlässlich einer Tagung des 15jährigen Bestehens der Hochschullernwerkstatt Erziehungswissenschaften in Halle im November 2020 entstanden.

Gefördert wurden die Tagung und der Band vom Zentrum für Lehrer *innenbildung, der Universitäts- und Landesbibliothek Sachsen-Anhalt sowie der Martin-Luther-Universität Halle-Wittenberg.

Dieser Titel wurde in das Programm des Verlages mittels eines Peer-Review-Verfahrens aufgenommen. Für weitere Informationen siehe www.klinkhardt.de.

Bibliografische Information der Deutschen Nationalbibliothek Die Deutsche Nationalbibliothek verzeichnet diese Publikation in der Deutschen Nationalbibliografie; detaillierte bibliografische Daten sind im Internet abrufbar über http://dnb.d-nb.de.

2020.k. (C) by Julius Klinkhardt.

Coverfoto: (C) Florian Johnke-Liese / Hochschullernwerkstatt.

Druck und Bindung: AZ Druck und Datentechnik, Kempten.

Printed in Germany 2020.

Gedruckt auf chlorfrei gebleichtem alterungsbeständigem Papier.

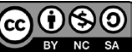

Die Publikation (mit Ausnahme aller Fotos, Grafiken und Abbildungen) ist veröffentlicht unter der Creative Commons-Lizenz: CC BY-NC-SA 4.0 International https://creativecommons.org/licenses/by-nc-sa/4.0/ 


\section{Inhalt}

Kathrin Kramer, Dietlinde Rumpf, Miriam Schöps und Stephanie Winter Einführung in den Band

\section{Teil 1: Historische Entwicklung}

Miriam Schöps und Dietlinde Rumpf

Universität mit Lernwerkstatt in Entwicklung -

Von der Materialausleihe zur Hochschuldidaktik

Hartmut Wenzel

Lernwerkstätten auch für die Sekundarstufen -

Zur Entwicklung der Hochschullernwerkstatt in Halle

Ulrike Stadler-Altmann, Susanne Schumacher, Enrico A. Emili,

Gerda Winkler und Elisabeth Dalla Torre

Hochschullernwerkstätten als Spielball der Bildungspolitik?

Die EduSpace Lernwerkstatt in der Südtiroler Lehrer*innenbildung zwischen nationalen und regionalen Bildungsinteressen

Hartmut Wedekind und Corinna Schmude

Von der Idee zum strukturell verankerten hochschuldidaktischen Prinzip -

Lernwerkstätten und Lernwerkstattarbeit im Studiengang „Erziehung und Bildung in der Kindheit" an der Alice Salomon Hochschule Berlin 68

\section{Teil 2: Wahrnehmung, Selbstverständnis, Einflüsse}

Dietlinde Rumpf und Corinna Schmude

NeHle - Internationales Netzwerk der Hochschullernwerkstätten -

Entwicklungsphasen einer Interessenvertretung und

eines gemeinsamen Begriffsverständnisses

Constantin Beyer und Florian Johnke-Liese

Hochschullernwerkstatt goes digital!

Alexandra und Michael Ritter

Drucken wie ,von gestern' in der Schule der Zukunft -

Die Schuldruckerei als Erfahrungsraum für Kinder und Studierende 
Livia Enders (geb. Makrinus) und Stephanie Winter

Studierende und Dozierende im Spannungsfeld zwischen

Theorie und Praxis - Die Hochschullernwerkstatt als Forschungsraum

John Marcus Sommer

Selbstwahrnehmung in der Hochschullernwerkstatt -

Wissenschaft zwischen Abstraktion und emotionalem Lernen

Lena Kliebe

Hochschullernwerkstatt als Raum und pädagogische Konzeption -

„Mein eigener Lernprozess“

Jerome Kampe

Geschichtsunterricht in der Lernwerkstatt!?

\section{Teil 3: Partizipation und Inklusion}

Melanie Schlag

Hochschullernwerkstatt schlägt Schlager

Kathrin Kramer

Nichtreformistische Reformen - Die Bedeutung von

Hochschullernwerkstätten auf dem Weg zu einer inklusiven Universität am Beispiel des Budgets für Arbeit

Johanna Ingenerf und Pascal Kurz

Menschenrechtsbasierte Bildung im internationalen Dialog -

Ein selbstorganisiertes Seminar mit Austausch

Ines Boban und Andreas Hinz

Hochschullernwerkstatt Halle als Möglichkeitsort -

Von WIRkstatt-Impulsen für inklusive Prozesse

Brigitte Kottmann und Alena Lensker (geb. Beckmann)

Die Lernwerkstatt und der Studiengang „Integrierte Sonderpädagogik“

an der Universität Bielefeld: Impulse, Synergien und Reflexionen

Sarah Dannemann, Tjark Neugebauer, Claudia Schomaker und Rolf Werning

Die LeibnizLernlandschaft: Diversität und Digitalisierung $\left(\mathrm{L}^{2} \mathrm{D}^{2}\right)$

gestalten - Konzeptionelle Gedanken für eine inklusive

Hochschullernwerkstatt an der Leibniz Universität Hannover 


\section{Teil 4: Reflexivität und Professionalisierung}

Kira Wybierek

Praxissemester in der Hochschullernwerkstatt -

Reflexion eines Lernprozesses

Eva Hoffart

„...da man lernt, eigene Gedanken und Ideen noch einmal zu vertiefen“ -

Theoretische Überlegungen und praktische Umsetzungen zum Reflektieren

von Lehramtsstudierenden

Edita Jung und Lena S. Kaiser

„Vielleicht romantisiere ich die Räume meiner Kindheit.“

Zugänge zu einer biographischen Reflexivität in

kindheitspädagogischen Hochschullernwerkstätten

Rolf-Torsten Kramer

Zum Problem der Professionalisierung im Lehramtsstudium und

zum Potenzial der Hochschullernwerkstatt

Mark Weißhaupt, Kathleen Panitz und Elke Hildebrandt

Die Inszenierung von „Theorie und Praxis“ sowie

„Neugier und Desinteresse“ bei der Professionalisierung

angehender Lehrpersonen in Hochschullernwerkstätten

Markus Peschel und Pascal Kihm

Hochschullernwerkstätten - Rollen, Rollenverständnisse und

Rollenaushandlungen

David Paulus, Patrick Gollub und Marcel Veber

Forschendes Lernen und Kasuistik: Überschneidungen und

Abgrenzungen bezogen auf Reflexivität in der

Hochschullernwerkstattarbeit

Pascal Kihm, Jenny Diener und Markus Peschel

Qualifizierungsprozesse und Qualifikationsarbeiten in

Hochschullernwerkstätten - Forschende Entwicklung einer

innovativen Didaktik 
10| Inhaltsverzeichnis

\section{Teil 5: Kooperation}

Georg Breidenstein, Sara Burkhardt, Thorid Rabe und Miriam Schöps

Zur Materialität des Lernens -

Anregungen aus einem interdisziplinären Forum

in der Hochschullernwerkstatt

Miriam Asmus, Kati Friebe, Mirjam Lewin und Kati Misselwitz

Entdeckendes Lernen und Digitale Medien - Ein Traumpaar .

Siglinde Spuller

Kooperation und Kooperatives Lernen als Prinzip

Hochschullernwerkstätten-adäquaten Lernens?

Eine konzeptionelle Verortung

Linda Balzer

Der Effekt selbstregulierenden und forschenden Lernens auf kooperative und individuelle Lernprozesse in der

Lernwerkstatt Religion Plural (LeRP)

Martin Lindner

Werkstattarbeit in der Biologiedidaktik -

Umstellung eines Praktikums auf Projektarbeit

Autor*innenbeschreibungen 
Livia Enders und Stephanie Winter

\section{„Das hatte ich mir anders vorgestellt" Studierende und Dozierende im Spannungsfeld zwischen Theorie und Praxis - Die Hochschullernwerkstatt als Forschungsraum}

\section{Zusammenfassung}

Wenn Studierende oder Lehramtsanwärter*innen zu ihrem Studium befragt werden, wird meist ein fehlender Praxisbezug reklamiert oder, noch mehr Praxis' eingefordert (vgl. SCHUBARTH or SPECK u. a. 2006, LIEBSCH 2010, MAKRINUS 2011alb). Dies ist erstaunlich, denn im Vergleich zu anderen Studiengängen zeigt sich im Lehramtsstudium ein großer Anteil berufsbezogener Elemente und integrierter Praxisphasen. Es scheint so, als sei der "Wunsch nach mehr Praxis" (MAKRINUS 2011b) eine Metapher für ein größer und diffuser angelegtes Problem (vgl. WERNET 2006, WERNET \& KREUTER 2007). Im folgenden Artikel soll deshalb den Fragen nachgegangen werden, wie es möglich sein kann, in Seminaren Theorie im universitären Wissenschaftskontext mit der eingeforderten Praxis zu verschränken. Was kann unter dem Begriff der Praxis subsummiert werden und inwiefern braucht es erst Praxis, oder stellen theoretische Reflexionen die Basis für jegliche Praxis dar und müssen deshalb zuerst vermittelt werden? Hierfür beziehen wir uns auf die Ergebnisse der biographieanalytischen Studie über die Verarbeitung und Reflektion des Studiums und seiner Praxisphasen aus der Perspektive von Lehramtsanwärter*innen in der Zweiten Phase der Lehrer*innenbildung (MAKRINUS 2011b) sowie die Reflexionen des Seminares „Zirkuspädagogische Ansätze im Lehrer*innenberuf aus Dozierendenperspektive“. Dazu wird zum einen anhand eines Fallbeispiels aus der biographieanalytischen Studie die rückblickende Bilanz einer Lehramtsanwärterin zu ihrem Studienweg rekonstruiert. Es ist ein Beispiel dafür, wie sich im Laufe des Studiums ein besonders schwieriges Verhältnis zur, Welt der Theorie' aufbauen kann und das Spannungsfeld von Theorie und Praxis als Legitimationsfigur für Aneignungsschwierigkeiten genutzt wird. Und andererseits schließt sich das Dilemma des Spannungsfeldes an, dass von Dozierenden erwartetet wird, einen , Wissens-und Könnensvorsprung'zu haben und Lernwege, Suchbewegungen inklusive Irrwege geduldig aushalten zu müssen. Nachfolgend plädieren wir für drei Aspekte, die unseres Erachtens nach eine zirkuläre Verzahnung von Theorie und Praxis ermöglichen: die Reflexion der eigenen biographischen Schul-Erfahrungen (1), reflektierte Praxis(selbst)erfahrungen (2) sowie Lernbegleitungen (3). 


\section{Der Wunsch nach mehr Praxis - der Fall Claudia Meinel}

In der folgenden Darstellung handelt es sich um Ergebnisse der Rekonstruktion eines berufsbiographischen Interviews mit einer Lehramtsanwärterin des Lehramts an Sonderschulen. Zum Zeitpunkt des Interviews ist Claudia Meinel seit einem Jahr Lehramtsanwärterin im Lehramt an Sonderschulen in den Fachrichtungen Lernbehinderten- und Körperbehindertenpädagogik. Sie hat das Sekundarschulfach Geschichte studiert und begann den Vorbereitungsdienst direkt nach dem Studium.

In ihrer Kindheit macht Claudia Meinel sehr positive Erfahrungen in der Körperbehindertenschule und mit Menschen mit Behinderungen. Ihr Vater wird selbst als ,körperbehindert' etikettiert und ist zugleich Lehrer an einer solchen Schule. Er nimmt seine eigenen Kinder häufig mit in die Schule, wodurch diese für Claudia Meinel zu einem sehr vertrauten, fast familiären Ort wird. Besonders beeindruckt ist sie von der Atmosphäre der wohlwollenden Anerkennung aller Beteiligten dieser Schule.

„(.) und das war (.) einfach ' $n$ tolles Klima (.) man is in diese Schule rein gekommen das war 'ne alte Villa (.) und die waren (.) die waren alle so freundlich miteinander die hatten so'n tollen Umgang //hm// (.)"1

Zugleich ist sie selbst, in ihrer Rolle als beobachtender Gast, kein volles Mitglied dieser Schule und sieht ihre eigenen, eher krisengeprägten Schulerfahrungen als schroffen Gegensatz hierzu. Aufgrund dieser positiven Erfahrungen in der Körperbehindertenschule absolviert Claudia Meinel dort verschiedene Praktika und begleitet Bekannte, die in diesem beruflichen Kontext arbeiten. Sie beobachtet wiederholt den angenehm freundlichen Umgang der Beteiligten und entwickelt den Wunsch, in irgendeiner Weise beruflich in dieses Setting involviert zu sein. Ihr Interesse fällt dabei eher auf therapeutische Berufe, der Lehrer*innenberuf ist zunächst noch wenig relevant. Welche genauen Tätigkeiten für sie als Person, mit ihren individuellen Fähigkeiten, in Frage kommen könnten, spielt bei ihrer Studienwahl keine Rolle. Die wichtigsten Dimensionen ihrer beruflichen Ausrichtung sind der Ort der Förderschule und der Wunsch, in irgendeiner Form über die Arbeit mit Menschen mit Behinderungen hier eingebunden zu sein. Claudia Meinel bewirbt sich für die Studienrichtungen Heilpädagogik und Lehramt an Sonderschulen. Da sie sich nicht auf einen konkreten Beruf festlegen kann, überlässt sie die Entscheidung über den Studiengang dem ,Zufall' und legt für sich fest, dass sie jenes Fach studiert, für das sie die erste Zusage erhält. Im weiteren Verlauf

1 Transkriptionsregeln der zitierten Passagen: Namen und Orte sind anonymisiert, keine Satzzeichen, (.) = Pause, Pkt. pro Sekunde; //hm// = Interviewerin; @.@ = lachen; man=wenn = schneller Anschluss, so = betont gesprochen, haben- = Wortabbruch 
des Interviews identifiziert sich Claudia Meinel vor allem mit der Vision eines zukünftigen Ziels. In ihm verankert sie den Motor für die Bewältigung der Studienanforderungen. Deutlich wird dabei, dass ihr das Studium als möglicher Bildungsraum fremd bleibt. Sie kann zu den theoretischen Inhalten keinen Zugang finden und empfindet das Arbeitspensum, das sie zu bewältigen hat, als sehr hoch. Sie gerät in eine tiefe Sinnkrise und überlegt, das Studium abzubrechen. Claudia Meinel führt die Ursachen für diese verhängnisvolle Entwicklung auf einen Bruch zwischen den Erwartungen an das Studium und den tatsächlich vorgefundenen Strukturen zurück, sozusagen eine Art Theorieschock. Sie entwickelt die Eigentheorie, dass sie „kein Theoriemensch“ (130) sei und somit den Anforderungen des Studiums notwendigerweise nicht gewachsen sein konnte. Das Problem ist dabei auf die institutionelle Ebene und den Unterschied von Theorie und Praxis verlagert. Die Universität ist dabei der institutionelle Ort der Theorie, an dem Praxis quasi nicht erwünscht ist. Gleichzeitig versucht Claudia Meinel diese Krise eigentheoretisch zu legitimieren.

\section{"Wir haben (.) 'n Haufen Theorie (.) und ich bin kein Theoriemensch absolut nich"}

Mit dem Ausdruck „'n Haufen Theorie“ werden die Inhalte des Studiums als unzugänglich, komplex und vor allem als zu viel markiert. Claudia Meinel findet keinen Zugang zu den Inhalten des Studiums und abstrahiert dies mit dem höheren Prädikat, „kein Theoriemensch“ zu sein. Dies impliziert zugleich, dass sie sich eher mit den praktischen Momenten der Ausbildung und der Arbeit in der Praxis definiert. Indem sie allerdings formuliert „kein Theoriemensch“ zu sein, hat sie lediglich ein Bild von dem, was sie nicht ist. Dies legt nahe, dass sie auch noch nicht fassen kann, ob sie dem Gegenhorizont des Theoriemenschen, nämlich dem Konstrukt einer kompetenten Praktikerin, entsprechen könnte. Die Eigentheorie bietet eine Entlastungsmöglichkeit zur Legitimation der Erfahrungen des Scheiterns im Bereich der Theorie. In dieser Konstruktion werden die Studienschwierigkeiten somit als grundsätzliche Nichtpassung zwischen ihrer Identitätskonstitution und den Anforderungen gewertet. Gleichzeitig wird ausgeschlossen, dass sie zwangsläufig vollständig ungeeignet für den Lehrerberuf sei. Denn potenziell besteht in dieser Figur die Möglichkeit, dass sie ihre Stärken im praktischen Bereich zur Geltung bringen kann, wobei sie sich darüber wiederum noch nicht vollständig im Klaren ist.

Die Praxis als Gegenhorizont zur unangenehmen Welt der Theorie wird für sie der antizipierte heilsame Ort, an dem sie erfahren kann, ob sie für den Lehrer*innenberuf wirklich geeignet ist. Diese Zielrichtung ist der zweite Motor, mit dem es ihr gelingt, sich den Anforderungen des Studiums trotz der massiven Krisen- und Belastungserfahrungen weiterhin zu stellen. Es ist zugleich eine Zielrichtung, die ihr die Öffnung für theoretische Inhalte versperrt. Trotz dieser Vorliebe für die Praxiselemente des Studiums reflektiert sie die obligatorischen Schulpraktika als 
zusätzliche Belastung. Für Claudia Meinel ist es ohnehin schwer, den Anforderungen des Studiums gerecht zu werden, wobei die Herausforderung in einem zusätzlichen praktischen Handlungsfeld ihre berufliche Eignung unter Beweis stellen zu müssen, sie zusätzlich unter Druck setzt. Es ist für sie von großer Bedeutung, inwiefern sie hier die Bekräftigung und Ermutigung durch signifikant Andere erfahren kann.

„(..) wie gesagt die Praktika wo man wo man einfach nur so (.) als Student angenommen wurde der sagt man kann sich ausprobieren die waren toll (.) die waren einfach Spitze //mb// da hat man auch'n Feedback bekommen von den Lehrern und die haben-gesagt haben (.) ja das geht so oder das geht so nich aus dem und dem Grund (.) und da lernt man auch was (.)"

Claudia Meinel erlebt solche Situationen als förderlich, in denen sie als entwicklungsbedürftige Lernende agieren kann. Wichtig ist dabei vor allem die Rolle der begleitenden Lehrer*innen. Sie stellt diese als Professionelle dar, die zwischen richtigen und falschen Handlungspraxen unterscheiden und diese modellartig vorstellen und begründen können. Auffällig ist, dass bei dieser Form des Arbeitsbündnisses ihre Rolle als Kollegin in den Hintergrund rückt. Sie sieht sich selbst als unterweisungsbedürftige Schülerin, die auch das Recht hat, Fehler zu machen, korrigiert zu werden und hierfür Begründungen zu erhalten. Es bedeutet in diesem Sinne die Anerkennung als Klient in der Krise (vgl. Oevermann 1996, 2002) und den Einbezug in die Institution Schule, mit welcher sie sich eher identifiziert als mit der Universität.

Kontrastierend stellt Claudia Meinel hierzu eine Form des kollegialen Bezugs dar, den sie als destabilisierend für die berufliche Entwicklung bilanziert.

„(.) und dann hatte man Kollegen dabei die in einem immer so (.) das gesehen haben der kommt frisch oder die kommt frisch aus der Uni die is mitten dabei die muss uns eigentlich was - (.) was erzählen können (.) und das macht keinen Spaß das is einfach 'ne Überforderung"(178-180)

Sie schildert eine Form des Kolleg*innenbezuges, in der ihr die Rolle als Innovatorin der Praxis (vgl. Radtke 2004) zugeschrieben wird. Hierbei wird sie als intensiv eingebundenes Mitglied der Institution Universität identifiziert, was ihrem eigenen Selbstbild massiv widerspricht. Sie wird als eine Vertreterin dieser Institution wahrgenommen, welche die Funktion hat, von neuen Impulsen und Veränderungen im pädagogischen Diskurs zu berichten:

„wenn man=wenn man dann von der Uni kommt man-man wird von den älteren Kollegen wirklich erstmal so (.) na ja sie haben doch jetz das neueste gelernt sie müssten's doch am besten wissen" 
Angesichts der eigenen Fremdheit gegenüber der Institution Universität und dem mit ihr verbundenen Konstrukt der Theorie fühlt sich Claudia Meinel dieser Situation nicht gewachsen. Sie erfährt dies als erneut destabilisierende Situation. Anstatt das Studium abzubrechen, lässt sich Claudia Meinel von Kommilitoninnen zur Teilnahme an einem Praxisprojekt überreden. Hier organisiert sie gemeinsam mit einer Kommilitonin über ein Schuljahr hinweg wöchentliche Lernangebote für eine Gruppe von Grundschüler*innen. Die Arbeit mit Grundschulkindern findet sie einerseits ,anstrengend“, leitet aus dieser Erfahrung andererseits für sich $a b$, dass ihre Entscheidung für den Sekundarschulbereich richtig war.

„(.) und ehm (.) was (.) was mich dann noch so bestärkt hat in meinem (.) in meinem Geschichtsstudium und in meinem Studium für (.) für die höheren Klassen (.) war dann ehm (.) diese Lernförderung (.) //m-hm// (.) bei Frau Lessing (.) weil das fand ich so anstrengend bei den Kleinen (.) dass ich gesagt hab or du hast dich so richtig @ entschieden@.@bei denGroßen arbeiten zuwollen"

Die Bekräftigung für das Weiterführen des Studiums zieht sie dementsprechend zunächst nicht aus dem Erleben gelingenden pädagogischen Handelns, sondern aus dem antizipierten Gegenentwurf, dass es mit einer anderen Altersgruppe weniger „anstrengend“ sein könnte.

Im Unterschied zu den herkömmlichen Praktika kann sie hier nicht auf unterstützende Kolleg*innen zurückgreifen, sondern muss sich selbst mit möglichen Wegen der Gestaltung von Lernanlässen auseinandersetzen. Sie beginnt, sich in die Kinder hineinzuversetzen und sich mit pädagogischen Fragestellungen zu beschäftigen. Diese Auseinandersetzung hilft ihr, ihre berufliche Zielorientierung zu verstärken und die hierfür notwendige Konfrontation mit ,Theorie' als notwendigem ,Muss' zu akzeptieren. Die ,Lernförderung' bleibt dabei ein Arbeitsschritt der Kompetenzaneignung auf dem Weg zum übergeordneten diffusen Ziel der ,Praxis'.

„(.) ja (.) aber es war eigentlich das Beste was ich machen konnte //hm/l (.) es war zwar viel Arbeit aber es war (.) es war Spaß (.) und es war ganz viel Praxis (.) und ganz viel gute Erfahrungen (..) von denen ich wie gesagt immer noch profitiere ja (.) I/hm//.) das allerbeste was passieren konnte doch (.) @.@ @ muss weiterlaufen (.)“

Das Praxisprojekt ist ein Teil der Abwicklung des institutionellen Ablaufmusters; ein Arbeitsschritt, in dem sie sich, auch aufgrund der praktischen Einbindung im Gegenhorizont der Theorie, eher aufgehoben fühlt. Claudia Meinel erfährt über die handlungsschematische Stützung ihrer Kommiliton*innen und der tatsächlichen Verbesserung durch die Inangriffnahme der ,Lernförderung' eine Stabilisierung ihrer krisenhaften Studiensituation. Sie findet ein labiles Gleichgewicht, mit dem es ihr möglich ist, das Studienziel weiterhin zu verfolgen. 
Der Übergang ins Referendariat wird aus der Perspektive Claudia Meinels insgesamt zu einer großen Befreiung von der „Qual“ (376) des Studiums. Sie freut sich, die "Theorie" hinter sich zu lassen und die Möglichkeit, nun Selbstwirksamkeit über praktisch handelndes Tätigsein zu erfahren.

Studierende, die wie Claudia Meinel nur schwer Zugänge zu einer wissenschaftstheoretischen Auseinandersetzung mit ihrem späteren Berufsfeld finden können, legen diese Schwierigkeiten den Lehrenden gegenüber selten offen. Dozierende können also davon ausgehen, dass es sie gibt, aber zumeist wissen die Lehrenden nicht, wer diese Student*innen sind oder worin konkret ihre Schwierigkeiten begründet liegen. Wie kann man nun versuchen, diesen Studierenden im universitären Kontext als Dozierende gerecht zu werden? Wie lassen sie sich einbinden in Seminargestaltungen und wie kann man ihnen die Möglichkeit eröffnen, auch als Nicht-Theoriemenschen Freude an der Auseinandersetzung mit ,n Haufen Theorie zu erleben?

\section{Zirkuspädagogische Ansätze - ein praxisorientiertes Seminar in der Hochschullernwerkstatt}

Wenn Claudia Meinel von sich selbst das Konstrukt des Nicht-Theoriemenschen aufwirft und somit gleichzeitig implizit den Gegenhorizont der Praktikerin, dann scheinen die beiden Begriffe der Theorie und Praxis Metaphern zu sein für die wissenschaftstheoretische Auseinandersetzung der Erziehungs- und Bildungswissenschaft sowie für Praxisanleitungen im Sinne von best practise Beispielen. Dass „sich das pädagogische Handeln nicht in der Anwendung rezeptförmiger Anweisungen erschöpft“ (vgl. KRÜGER 2019, 13), begründet sich in drei Argumenten: in der Vielzahl pädagogischer Konzepte und zum Teil kontroverser erziehungswissenschaftlicher Perspektiven (1), in der Einzigartigkeit der Situationen und der Individualität der Menschen, die im pädagogischen Kontext miteinander interagieren (2) sowie in der Notwendigkeit, pädagogisches Wissen auf zukünftige Situationen zu transformieren und weiterentwickeln zu können (3). Zur pädagogischen Handlungskompetenz gehören demnach eine kritische Auseinandersetzung mit vorliegenden Positionen sowie eine immerwährende Reflexion des eigenen pädagogischen Handelns (vgl. ebd., 14). Die theoretische Wissensvermittlung im universitären Kontext ist somit als Ermöglichung einer pädagogischen Handlungskompetenz zu verstehen.

Um den Begriff der Praxis bzw. des Praxisbezuges zu schärfen, muss an dieser Stelle eine Abgrenzung zu unterschiedlichen Perspektiven auf Praxis vorgenommen werden. Unter der Herstellung von Praxisbezügen fasst Hedtke Formen wie „... eigener Unterricht, Hospitationen, empirische Unterrichtsforschung, 
Simulationen, Interviews mit Praktikern oder Lernenden, Planung von Unterricht, Reflexion von Fallstudien (Video, Text) oder Diskussion von Texten und Daten über Praxisaspekte“ zusammen (vgl. ebd. 2003, 10). Perspektiven auf die Praxis (angehender) Lehrer*innen sind „in erkennender (Theorie), handelnder (Pragmatik) oder reflektierender (Kommunikation) Absicht möglich. Isoliert man berufspraktisches Handeln von Reflexion, wird es auf instrumentelles Handeln verkürzt (Technik)“ (vgl. ebd.), da sie eine kritische Distanz zum eigenen Handeln erst ermöglichen. Somit sind Perspektiven auf Praxis als wissenschaftstheoretische Auseinandersetzung - als "Theorie“ - zu verstehen, die ein Nachdenken über Praxis als ureigenes Forschungsfeld beinhaltet und umgekehrt werden in der Praxis eigene Theorien über Praktiken, Praxisbezüge, Praxisrelevanz, Anwendbarkeit und Umsetzbarkeit etc. gebildet.

Ausgehend von diesen Setzungen lag dem Blockseminar „Zirkuspädagogische Ansätze im Lehrer*innenberuf“ am Institut für Rehabilitationspädagogik der Martin-Luther-Universität Halle-Wittenberg eine Konzeption zugrunde, die sowohl eine wissenstheoretische Vermittlung vornimmt, als auch die Erprobung von Praktiken initiiert sowie Praxisbezüge herstellt. Die Studierenden sollten zunächst eine vertrauensvolle Atmosphäre und die Begeisterung der Dozentin am Lerngegenstand erfahren, um sich darauf aufbauend anhand eigener Fragestellungen einen wissenschaftstheoretischen Zugang zu verschiedenen Elementen der Zirkuspädagogik zu erschließen. Eine kritische Distanz zum eigenen Lernweg sollte mittels Lernbegleitungen initiiert werden.

Die Hochschullernwerkstatt wurde hierfür als Ort gewählt, da sie entdeckendes und forschendes Lernen ermöglicht (vgl. Schneider 2008, 5) und die Studierenden im Tandem so wechselseitig die Rolle der Lernenden und Lehrenden einnehmen konnten. Die Hochschullernwerkstatt regt als Ort eine zirkuläre Verzahnung von Theorie und Praxis im je eigenen Tempo der Lernenden an und schafft es, so „die universitäre Logik auf eine gesunde Weise aus(zu)hebel(n)“ (vgl. WybiereK in diesem Band).

Mit einem gemeinsamen spielerischen Einstieg erfuhren die Teilnehmer*innen zugleich eine von drei Teilkomponenten der Jonglage: die emotionale Komponente. Sie beinhaltet die Gründe, warum jemand jongliert. Die Freude an der Bewegung, an der Handlung des Jonglierens motiviert dazu, seine Kreativität zum Ausdruck zu bringen und sich mehr Können und Wissen aneignen zu wollen (vgl. Oberschachtsiek 2003). Diese Bewegungsfreude können Lehrende nutzen: „Easy stuff, nice to do!“ (Bотteri 2019, 6). Einen Ball und einen Ring wieder und wieder von einer Hand in die andere zu werfen, vermittelt auf Dauer keine Freude, sondern langweilt. 

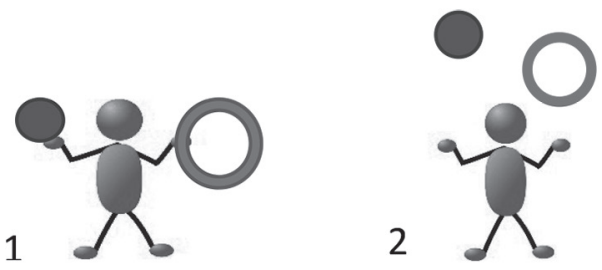

3

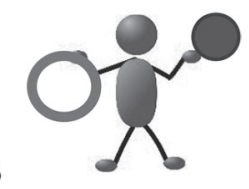

Abb. 1

Wird stattdessen eine Geschichte erzählt, transportiert dies nicht den analytischen Weg, der zwar ausgeführt wird, sondern der Spaß am Tun rückt in den Fokus der Aufmerksamkeit. So kann beispielsweise der Ball eine schöne runde Kuh symbolisieren und der Reifen den Mond. Die Kuh versucht nun, über den Mond zu springen und, wenn der Ball dabei runterfällt, dann hat die Kuh den Fehler gemacht und nicht der*die Lernende.

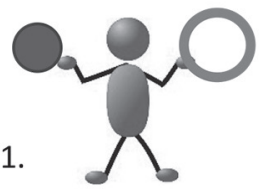

2.
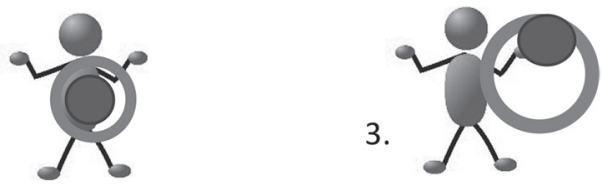

Abb. 2

Ebenso kann die Kuh durch den Reifen springen. Bei beiden Übungen wird der Handwechsel geübt, der für das Jonglieren Lernen essentiell ist. Jedoch werden die zweite und dritte Teilkomponente des Jonglierens (die technische Komponente - also das WIE der Handlung, damit drei Bälle zu einer organisierten Form in die Luft geworfen werden können - und die kognitive Komponente - also das WANN und WO einer Handlung, die angibt, zu welcher Zeit an welchem Ort etwas passiert) nicht direkt vermittelt, sondern in verschiedenen, abwechslungsreichen Variationen - und dennoch muss die Technik genau beobachtet werden, sonst funktioniert das Spiel nicht.

Die sich daran anschließende wissenschaftstheoretische Erarbeitung setzte sich aus entwicklungspsychologischen, didaktisch-methodischen und fachwissenschaftlichen Inhalten zusammen.

Die entwicklungspsychologische Perspektive basierte im Wesentlichen auf der Theorie der Zone der nächsten Entwicklung (vgl. WYGotzKI, 1987). d. h. es galt zu klären, aufgrund welcher Angebote Lernende die Linie des Nicht-Jonglieren-Könnens zum Jonglieren-Können ohne bzw. mit wenig Stress überschreiten. Objekte mit unserem Körper zu bewegen, ist unser Alltag. Das Jonglieren als zeitlich geordnete und koordinierte Bewegung von Objekten (vgl. BоттеRI 2019, 
4) erfordert dabei lateral synchrone, lateral asynchrone, bilateral synchrone und bilateral asynchrone Bewegungen (vgl. ebd.), die im spielerischen Ausführen die Bewegungskoordination fördern sowie die Hirnaktiviät in hohem Maße anregen (vgl. MaX-Planck-Gesellschaft 2010).

Die fachwissenschaftliche Perspektive erarbeitet die Technik der Jonglage an sich:

\begin{tabular}{lll}
\hline Zeit & Bewegung & Ort \\
\hline synchron & gemeinsam & lateral \\
asynchron & unterschiedlich & bilateral \\
\hline
\end{tabular}

Dabei lassen sich drei Typen von bilateralen Bewegungen unterscheiden

- beide Hände werfen und fangen

- es wird von einer Hand in die andere geworfen, wobei die Körpermitte nicht überkreuzt wird

- beide Hände werfen in die jeweils andere Hand unter Nutzung der zeitlichen Komponente, da asynchrone Bewegungen einfacher auszuführen sind.

Die didaktisch-methodische Perspektive wendet den Blick ab vom bisherigen linearen Erlernen des Jonglierens im Sinnes eines Mehr an zu jonglierenden Objekten, da dies schnell zu Frustration und Misserfolgen führen kann, hin zu einem Umdenken: Wie gelangt man vom Einfachen zum Schweren? Dafür benötigt es zunächst ein Konzept vom Handlungsablauf des Jonglierens, sodass Aktivitäten zunächst im zweidimensionalen Bereich angeboten werden. Dazu eignet sich beispielsweise das Rollen von drei Bechern auf einem Tisch. Die Tischfläche muss leicht in Schräglage arrangiert sein, indem man etwa Bücher unter zwei Tischbeine stellt. Die Becher werden zunächst in die Ausgangsposition (Abb. 1) gestellt und dann nacheinander mit der offenen Seite abwechselnd nach außen gelegt (Abb. 2 bis Abb. 4). Durch die Neigung der Tischfläche sowie aufgrund der kegelförmigen Beschaffenheit der Becher rollen sie jeweils nach links oder rechts unten (Abb. 5 und 6). Je höher der Neigungswinkel des Tisches, desto schneller rollen die Becher. Nach mehreren Durchläufen kann man die Becher wieder in die Ausgangsposition stellen. Der Vorteil an dieser Übung besteht darin, dass die Reihenfolge der auszuführenden Bewegungen verlangsamt und wie auf einem Bild sichtbar gemacht wird. 


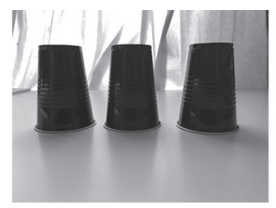

(1)

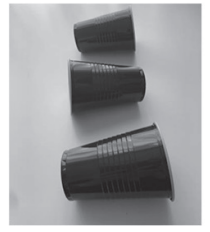

(4)

(5)

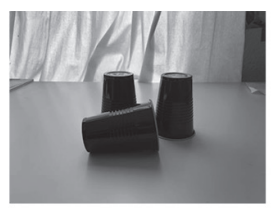

(2)

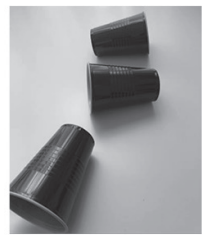

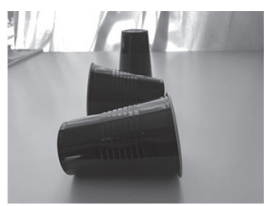

(3)

Abb. 3: Eigenes Bild. Idee: Craig Quat@quatrops.de

Erst wenn sich dieses Verständnis ausgebildet hat, erfolgt das Zerlegen eines komplexen Bewegungsablaufes in sequenzierte Einheiten im dreidimensionalen Bereich, damit das Gehirn es schafft, die Bewegungsausführung zu veranlassen. So kann beispielsweise in Partner*innenübungen das Fangen und Werfen mit mehreren Objekten geübt werden, wobei die Partner*innen zu einem Zeitpunkt entweder fangen oder werfen. Erst danach geht man zum Jonglieren mit mehreren Objekten über.

Fragestellungen, die darauf aufbauend in einem dynamischen Prozess gefunden und formuliert wurden, spiegelten sowohl die Vielfalt des Jonglierens und der Zirkuspädagogik als auch eigene (biografische) Erfahrungen der Studierenden wider. So setzten sich einige Studierende mit dem Zusammenhang der Beschaffenheit von Bällen und ihrem Einfluss auf das Erlernen des Jonglierens auseinander, nachdem sie beim eigenen Versuch des Jonglierenlernens mit einer bestimmten Art von Bällen deutlich bessere und schnellere Ergebnisse erzielen konnten. Andere suchten nach Optimierungsmöglichkeiten für das Juggleboard (QUAT o.A.), da sie in vorangegangenen Schulpraktika erleben mussten, dass Kinder aufgrund ihrer körperlich-motorischen Beeinträchtigung nicht an allen Aktivitäten partizipieren konnten und sie anhand der Fragestellung nach einer Möglichkeit des Jonglierenlernens für alle Kinder suchen wollten. Diese Lernwege zeigten sich also als „Schnittstellen individueller biographischer Entwürfe, sozialer Interaktionen und gegenständlicher Strukturen“ (vgl. GEILING 2000, 41). Die Lernbegleitungen der Studierenden erfolgten mittels Gesprächen, insofern Bedarf danach geäußert beziehungsweise Gesprächsangebote angenommen wurden. Interaktionen dieser Art setzen die Selbstregulationsfähigkeit der Lehrenden und Lernenden ebenso wie ein aktives Zuhören und Verstehen voraus, um „Wissen und Lernen während 
des Lernprozesses in Worte fassen und den eigenen Lernprozess reflektieren zu können" (vgl. Puhr \& Winter 2019, 38).

Sobald man sich für einen Weg entscheidet, schließt man damit zugleich andere Wege aus - was nicht heißen soll, dass man sie nicht zu einem anderen Zeitpunkt gehen kann. Für die aktuelle Situation hat man jedoch eine Auswahl getroffen. $\mathrm{Ob}$ man als Dozierende mit der Entscheidung für diese Art der Konzeption Studierenden wie Claudia Meinel gerecht werden kann, wird erst im Handeln sowie im (gemeinsamen) Reflektieren der Lernwege sichtbar. Ob man diesen Weg der Wissensvermittlung ein weiteres Mal gehen wird, ihn leicht adaptiert oder einen gänzlich anderen Weg geht, zeigt sich auch für Dozierende in der Lernbegleitung.

\section{Die Hochschullernwerkstatt als Forschungsraum}

Im Folgenden soll nun der Frage nachgegangen werden, inwieweit die Hochschullernwerkstatt einen Ort darstellen kann, um ein Nachdenken über schulische Praktiken anzuregen, also eine subjektive Theorie von der Praxis zu entwerfen, diese zu reflektieren, um so eigene Bezüge zu wissenschaftstheoretischen Positionen herstellen zu wollen und zu können. Biographisches Lernen/biographische Arbeit, die Reflexion der eigenen Schul-Erfahrungen sowie Lernbegleitungen stellen unseres Erachtens hilfreiche Formen der Unterstützung in diesem Prozess dar. Eine Hochschullernwerkstatt kann als konkreter universitärer Ort verstanden werden, der durch eine Fülle an Material als ,anregende Lernumgebung“ fungiert (Ernst \& Wieneke-Kranz 1987 zit. n. Pallasch \& Reimers 1990, 85). Gleichzeitig ist es die Art und Weise der Auseinandersetzung in und mit diesem Raum, welche die Hochschullernwerkstatt als solche charakterisiert (vgl. Pallasch \& ReIMERS 1990). Es geht darum, sich einem Gegenstand aktiv zuzuwenden und diesen zu bearbeiten, wobei der verfügbare Material- und Werkfundus Anregungen geben kann und die mit einem Gegenstand verbundene Fragestellung gleichzeitig auch weiterzuentwickeln. Im Gegensatz zum Workshop wird in dieser, Werkstatt' das Zusammenarbeiten von ,Nicht-Expert*innen' an einer gemeinsamen Zielsetzung mit dem Zweck des Ausgleichs und der Ergänzung unterschiedlicher Kompetenzen praktiziert (vgl. ebd.). Es bedeutet, ,in Dialog zu treten mit einer Sache, einem Thema, einer Frage, d.h. Erfahrungen an einem Gegenstand zu machen" (Bolland 2011, 132). Die Lernwege und Strategien sind dabei offen und unbestimmt, entstehen im gemeinsamen Agieren und werden im Zuge der gemeinsamen Reflexion greifbar. „Der Lernanstoß wird indirekt ausgelöst, z. B. durch konsequente Bearbeitung eines persönlichen Themas, durch versuchsweise Auseinandersetzung mit einer Sache, durch eine irritierende Phänomenbetrachtung, eine poetische Bearbeitung, eine Situationsverfremdung." (ebd. 132). Die- 
ses Konzept eröffnet vielseitige Möglichkeiten, einen zirkulären Prozess sich abwechselnder und sich wechselseitig bedingender Elemente von Theorie und Praxis zu konstituieren: biographische Selbsterfahrungen, reflektierte Praxis(selbst)erfahrungen sowie Lernbegleitungen sind hier beispielhaft aufgerufen. Die Hochschullernwerkstatt bietet hierfür den Raum zur Verwirklichung eigener Ideen und der Entdeckung kreativer Potenziale. Dabei findet gleichzeitig eine Prozessreflexion (vgl. Pallasch \& Reimers 1990) statt, die vor allen Dingen stärkenorientiert biographische Erfahrungen und Praxis(selbst)erfahrungen aufgreift. Mögliche Fragen für biographische Arbeit in der Lehrer*innenbildung könnten sein:

- Welche Bezugspersonen haben mich geprägt? Wie gestaltete sich die Beziehung mit ihnen?

- Welche Erfahrungen habe ich mit ,Schule‘ gemacht?

- Welche Erfahrungen habe ich mit ,Lernen' gemacht?

- Worin liegen meine Ängste bzgl. der Studien- bzw. Berufswahl?

- Worin liegen meine persönlichen Ressourcen und Unterstützerquellen?

- Worin fühle ich mich kompetent?

- Welche Erwartungen habe ich an meine berufliche Zukunft?

- Wie sind diese Erwartungen mit meinem bisherigen Werdegang verknüpft?

- Wie nehme ich Lernsituationen wahr? Wie gehe ich Probleme und Aufgaben an?

- Welche Konzepte habe ich von Theorie und Praxis, von Wissenschaft und Schulpraxis?

Diese explizite Auseinandersetzung mit der Biographie bedarf einer ganz sensiblen Betreuung und muss unbedingt im Kontext der Vertraulichkeit und Freiwilligkeit behandelt werden. Es stellt sich die Frage, ob diese Form der Auseinandersetzung im Rahmen des Lehramtsstudiums überhaupt möglich ist, da die Bewertungszwänge im Studium implizit immer erhalten bleiben. Neben dieser expliziten Arbeit an der eigenen Biographie bietet sich daher die Auseinandersetzung mit Biographien Anderer als mögliche Auslöser eigener biographischer Selbstreflexionen an. Der Unterschied besteht hier darin, dass den fremden Biographien mit einer schützenden Distanz begegnet werden kann, sie gleichzeitig aber Identifikations- und Distanzierungsdimensionen enthalten. Auch hier ist ein sensibler Umgang mit den lebensgeschichtlichen Materialien gefragt. Gerade die Vieldeutigkeit und Ungewissheit biographischer Entwicklungen herauszuarbeiten, ist ein Ziel. Gleichzeitig sollte aber auch betrachtet werden, in welcher Art und Weise strukturelle Bedingungen, wie gesellschaftliche Rahmenstrukturen und individuelle Verarbeitungs- und Bewältigungsmuster die lebensgeschichtlichen Entwicklungsprozesse beeinflussen.

Wie Studierende die eigene Schulzeit erlebt haben, welche Erfahrungen aus schulischen Praktika sie mitbringen, beeinflusst ihr eigenes, späteres Lehrer*innen- 
handeln. Reflexion als eine häufig postulierte Kernkompetenz von Lehrer*innen (vgl. Wyss 2008) sollte demnach auch das Bewusstmachen der eigenen Schulerfahrungen beinhalten. Wie habe ich mich in meiner eigenen Schulzeit gefühlt? Gab es Lieblingslehrer*innen und warum waren es gerade diese? Welche Lehrer*innen haben mir Angst gemacht und welche Gründe gab es dafür? Was habe ich für meine eigene berufliche Zukunft aus dieser Zeit mitgenommen? Welche Erfahrungen habe ich bereits in Schulpraktika gesammelt? Welche Rolle habe ich während dieser Zeit eingenommen und welche wurde mir zugeschrieben? Dies sind beispielhafte Fragen, um eine kritische Distanz zur eigenen Schulzeit und zu eigenen Schulerfahrungen zu erlangen.

Einen weiteren Aspekt, der als Anregung für Studierende und Dozierende gleichermaßen verstanden werden kann, um sich in einem zirkulären Prozess Wissen und Können anzueignen, bieten Lernbegleitungen innerhalb von Seminaren. „Die Bedeutung von Reflexion und Reflexivität wird besonders dort herausgestellt, wo eine grundsätzliche kategoriale Verschiedenheit und Eigenlogik von Wissen einerseits und Können andererseits bzw. von Theorie und Praxis postuliert wird" (vgl. Neuweg 2011, zit. n. Degeling et al. 2019, 85). Dieses Nachdenken über eigene Praktiken kann in gemeinsamen Lernprozessbegleitungsgesprächen stattfinden, um eine enge Begleitung und regelmäßige kritische Auseinandersetzung mit dem Erlebten überhaupt zu ermöglichen. Dies kann in Form von Gesprächen stattfinden, insofern diese Gespräche als offene Angebote verstanden werden, die angenommen werden können aber nicht müssen, die zu einer kritisch-produktiven Auseinandersetzung anregen, ohne den Eroberungsprozess zu verlangsamen oder gar zu hemmen. Diese Dialoge orientieren sich in erster Linie an den Bedürfnissen der Lernenden und nicht an einem vorstrukturierten Gesprächsleitfaden. Das oben beschriebene Seminar "Zirkuspädagogik in der Lehrer*innenbildung“ stellte das Ergebnis einer Suche nach Möglichkeiten der Theorieaneignung über einen praxisorientierten Zugang dar. Die Lernbegleitungen der Studierenden auf ihren Lernwegen bildeten einerseits einen möglichen Zugang zur Theorieaneignung seitens der Studierenden und andererseits stellte es die Dozentin vor die Herausforderung, gleichzeitig als Modell für die Lernenden zu fungieren, was bei konsequenter Durchführung ebenfalls eine Lernbegleitung und Reflexion des eigenen Lehrendenhandelns und der eigenen Praktiken nach sich zieht (vgl. RumpF \& SсHӧрs 2017, 97f).

\section{Ausblick}

Insgesamt betrachtet, wird die Hochschullernwerkstatt als ein Ort konzipiert, der offene Lernwege ermöglicht, aktivierende Handlungsanforderungen bereitstellt und zugleich die Möglichkeit eines gleichwertigen Unterstützer*innenkreises eröffnet. Inwiefern diese Wege und Möglichkeiten einerseits durch Dozierende eröffnet und andererseits durch Studierende als solche auch angenommen und 
positiv erfahren werden und reflexiv tatsächlich Veränderungen hervorrufen, ist die wesentliche Frage. Es ist denkbar, dass die Hochschullernwerkstatt seitens der Lehrenden lediglich als ein Raum angesehen wird, in dem viele Materialien versammelt und frei zugänglich sind oder als einfache Möglichkeit, veränderte Sitzformationen herstellen zu können oder auch als explizites Anschauungsobjekt. Ebenso ist es sehr wahrscheinlich, dass auch hier Aneignungsprobleme seitens der Lernenden auftreten, dass die Offenheit als nicht zu bewältigende Herausforderung erlebt wird, dass sich der intendierte gemeinsame Austausch auf oberflächliches ,Rauschen' reduziert, dass die Ergebnisse sehr wohl vorhersehbar bleiben und ,Lernimpulse' als Arbeitsaufgaben abgearbeitet werden.

Die Hochschullernwerkstatt bietet viele Möglichkeiten. Ob und wie sie genutzt werden, hängt von den handelnden Akteur*innen selbst ab, ist aber auch eine Frage der organisatorischen Einbettung: ist der Besuch und die Auseinandersetzung in und mit der Hochschullernwerkstatt zeitlich im Studienrahmen eingeplant, ist die Zeit in der Hochschullernwerkstatt gleichzeitig ein freiwilliges Angebot, gibt es Wahlmöglichkeiten, werden konzeptuelle Veränderungen in studentischer Partizipation geplant und umgesetzt?

Grundsätzlich ist allerdings zu bedenken und auch im Diskurs mit den Studierenden offen zu legen, dass auch die Hochschulbildung einem grundsätzlichen „Technologiedefizit“ unterliegt (Luhmann \& SCHORR 1982). Die Lernwerkstatt ist nicht frei von strukturellen Zwängen und Paradoxien. Somit ist die Dynamik von Lern- und Entwicklungsprozessen auch innerhalb dieses frei und offen angelegten Kontextes immer als kontingent und ungewiss zu beschreiben. „Eine Aufgabe der Hochschullernwerkstatt ist es, die Universität zu irritieren“ (vgl. WyBIEREK in diesem Band). Sind Dozierende und Studierende also gleichermaßen bereit, fernab von zu erwartenden oder zu erbringenden Leistungen, sich einzulassen auf offene Lernwege und entdeckendes Lernen, neugierig zu sein, sich mit einem Thema von besonderem Interesse in den Bann ziehen zu lassen, Fragen zu stellen und zu hinterfragen ... kurz: sind Studierende und Dozierende bereit, sich einzulassen auf einen reflexiven Forschungs- und Lernprozess als wechselseitig Lehrende und Lernende? Dann bieten Lernwerkstätten an Hochschulen und Universitäten diesen Raum!

\section{Literatur}

Bolland, Angela (2011): Forschendes und biographisches Lernen. Das Modellprojekt Forschungswerkstatt in der Lehrerbildung. Bad Heilbrunn: Klinkhardt.

BOTTERI, Lapo (20I9): Study of the effects of Juggling sessions with the juggleboard on subjects with intellectual disability. Im Internet: https://www.quatprops.net/lapo-paper.

Geiling, Ute (2000): Integrative Pädagogik mit Kindern in erschwerter Lernsituation - oder: Für eine Pädagogik, die Kinder stark macht. In: Geiling, Ute (Hrsg.): Pädagogik, die Kinder stark macht. Ansätze zur Arbeit mit Kindern in Not. Opladen: Leske Budrich, 41-58. 
Grunert, Cathleen (1999): Vom Pionier zum Diplom-Pädagogen: Lebensgeschichten und Berufsperspektiven von ostdeutschen Studierenden im Diplomstudiengang Erziehungswissenschaft. Opladen: Leske Budrich.

Hattie, J. A. C. (2009): Visible Learning. A synthesis of over 800 meta-analyses relating to achievement. London \& New York: Routledge.

Hedtke, Reinhold (2000): Das unstillbare Verlangen nach Praxisbezug. Zum Theorie-Praxis-Problem der Lehrerbildung am Exempel Schulpraktischer Studien. Im Internet: : https://www.uni-bielefeld. de/soz/ag/hedtke/pdf/praxisbezug_lang.pdf.

Hölzle, Christina \& JAnSEn, Irma (Hrsg.) (2011): Ressourcenorientierte Biografiearbeit. Grundlagen-Zielgruppen-Kreative Methoden. Wiesbaden: Springer.

KrÜGER, Heinz-Hermann (2019): Erziehungs- und Bildungswissenschaft als Wissenschaftsdisziplin. Opladen: Budrich.

Liebsch, Katharina (Hrsg.) (2010): Reflexion und Intervention. Zur Theorie und Praxis Schulpraktischer Studien. Hohengehren: Schneider.

Luhmann, Niklas \& Schorr, Karl E. (1982): Das Technologiedefizit der Erziehung und die Pädagogik. In: dies.: Zwischen Technologie und Selbstreferenz. Fragen an die Pädagogik. Frankfurt/Main: Suhrkamp, 11-40.

Makrinus, Livia (2011a): Praktika im Lehramtsstudium - qualitative Einblicke in biographische Relationierungsprozesse. In: Zeitschrift für Grundschulforschung. Bildung im Elementar- und Primarbereich. Jg.4, Heft 2, 111-124.

Makrinus, Livia (2011b): Der Wunsch nach mehr Praxis. Lehramtsanwärter im Spannungsfeld von Theorie und Praxis. Qualitative Einblicke in die biographische Relationierung von Schulpraktika und Praxiserfahrungen während des Vorbereitungsdienstes. Dissertation. Martin-Luther-Universität Halle-Wittenberg.

Makrinus, Livia \& Klektau, Claudia (2019): Praxisbezug in der Lehrer*innenbildung. Möglichkeiten und Grenzen einer biografischen Entwicklungsbegleitung am Beispiel des Schüler*innenhilfeprojektes Halle. In: Bartusch, Steffen; Klektau, Claudia; Simon, Toni; Teumer, Stefanie \& Weidermann, Anne (Hrsg.): Lernprozesse begleiten. Anforderungen an pädagogische Institutionen und ihre Akteur*innen. Wiesbaden: Springer, 105-114.

Max-Planck-Gesellschaft (20 Io): Neuronale Plastizität. Im Internet: https://www.mpg.de/21486/ Neuronale_Plastizitaet.

Meister, Gudrun (2005): Das unterrichtliche Selbstverständnis von LehrerInnen. Empirische Muster im Kontext von Unterricht und Biographie. Wiesbaden.

Miethe, Ingrid (2011): Biografiearbeit. Lehr- und Handbuch für Studium und Praxis. Weinheim, München.

OвERschachtsiek, Bernd (2003): Jonglieren und Mehr ... Handbuch Bewegungskünste für Schule, Verein und Freizeit.

Oevermann, Ulrich (1996): Theoretische Skizze einer revidierten Theorie professionellen Handelns. In: Combe, Arno \& Helsper, Werner (Hrsg.): Pädagogische Professionalität. Frankfurt/Main: Suhrkamp, 70-183.

Oevermann, Ulrich (2002): Professionalisierungsbedürftigkeit und Professionalisiertheit pädagogischen Handelns. In: Kraul, Margret; Marotzki, Winfried \& Schweppe, Cornelia (Hrsg.): Biographie und Profession. Bad Heilbrunn: Klinkhardt, 19-64.

Pallasch, Waldemar \& Reimers, Heino (1990): Pädagogische Werkstattarbeit. Eine pädagogisch-didaktische Konzeption zur Belebung der traditionellen Lernkultur. Weinheim, München: Juventa.

Puhr, Kirsten \& Winter, Stephanie (2019): Lern(prozess)begleitungen als unterrichtliche Interaktionen. In: Bartusch, Steffen; Klektau, Claudia; Simon, Toni; Teumer, Stephanie \& WeiderMANN, Anne (Hrsg.) (2019): Lernprozesse begleiten. Anforderungen an pädagogische Institutionen und ihre Akteur*innen. Wiesbaden: Springer, 33-46. 
QuAT, Craig (o.A.): The Juggle Board. A practical guide. Im Internet: https://www.quatprops.net/ guidebook-engligh.

RadtKe, Frank-Olaf (2004): Der Eigensinn pädagogischer Professionalität jenseits von Innovationshoffnungen und Effizienzerwartungen. Übergangene Einsichten aus der Wissensverwendungsforschung für die Organisation der universitären Lehrerbildung. In: КосH-Priewe, Barbara; Kolbe, Fritz-Ulrich \& WILDT, Jochen (Hrsg.): Grundlagenforschung und mikrodidaktische Reformansätze zur Lehrerbildung. Bad Heilbrunn: Klinkhardt, 99-149.

ReH, Sabine (2003): Berufsbiographische Texte ostdeutscher Lehrer und Lehrerinnen als „Bekenntnisse“. Interpretationen und methodologische Überlegungen zur erziehungswissenschaftlichen Biographieforschung. Klinkhardt: Bad Heilbrunn.

Rumpf, Dietlinde \& Sснӧps, Miriam (2017): Reflexion als Gegenstand qualitativer Forschung in der Arbeit der Lernwerkstatt Erziehungswissenschaften/Halle. In: Franz, Eva-Kristina; GunzenraINer, Johannes; Wedekind, Hartmut; Müller-Naendrup, Barbara \& Peschel, Markus (Hrsg.): Lernen und Studieren in Lernwerkstätten. Impulse für die Theorie und Praxis. Bad Heilbrunn: Klinkhardt.

SchneIder, Siegfried (2008): Vorwort. In: Bayerisches Staatsministerium für Unterricht und Kultus (Hrsg.): Über die Hand zum Verstand. Handreichung für den Aufbau einer Lernwerkstatt. München, 5.

Schubarth, Wilfried; Speck, Karsten; Grosse, Ulrike; Seidel, Andreas \& Gemsa, Charlotte (2006): Die zweite Phase der Lehrerbildung aus Sicht der Brandenburger Lehramtskandidatinnen und Lehramtskandidaten. Die Potsdamer LAK-Studie 2004/5. In: Schubarth, Wilfried \& Pohlenz, Philipp (Hrsg.): Qualitätsentwicklung und Evaluation in der Lehrerbildung. Die zweite Phase: Das Referendariat. Potsdamer Beiträge zur Lehrevaluation. Potsdam: Universitätsverlag, 13-177.

Tiefel, Sandra (2004): Beratung und Reflexion: eine qualitative Studie zu professionellem Beratungshandeln in der Moderne. Wiesbaden: Springer.

Wernet, Andreas \& Kreuter, Vera (2007a): Lehrerbildung als Identitätsproblem: Fallstudien zu Problemen der subjektiven Selbstverortung von ReferendarInnen. Abschlussbericht einer vom Zentrum für Lehrerbildung der Universität Potsdam geförderten hermeneutischen Begleituntersuchung zur Potsdamer LAK-Studie. Potsdam: Universitätsverlag.

Wernet, Andreas \& Kreuter, Vera (2007b): Endlich Praxis? Eine kritische Fallrekonstruktion zum Praxiswunsch in der Lehrerbildung. In: Schubarth, Wilfried; Speck, Karsten \& Seidel, Andreas (Hrsg.): Endlich Praxis! Die zweite Phase der Lehrerbildung. Potsdamer Studien zum Referendariat. Frankfurt/Main: Peter Lang, 183-196.

WERnET, Andreas (2006): „Man kann ja sagen, was man will: es ist ein Lehrer-Schüler-Verhältnis.“ Eine fallanalytische Skizze zu Kollegialitätsproblemen im Referendariat. In: Sснивarth, Wilfried \& Pohlenz, Philipp (Hrsg.): Qualitätsentwicklung und Evaluation in der Lehrerbildung. Die zweite Phase: Das Referendariat. Potsdamer Beiträge zur Lehrevaluation. Potsdam: Universitätsverlag, 193-207.

WybiereK, Kira (2020): Praxissemester in der Hochschullernwerkstatt - Reflexion eines Lernprozesses. In diesem Band.

Wygotsкi, Lew (1987): Ausgewählte Schriften, Bd. 2: Arbeiten zur psychischen Entwicklung der Persönlichkeit. Köln: Pahl-Rugenstein (Orig. Moskau 1956).

Wyss, Corinne (2008): Zur Reflexionsfähigkeit und -praxis der Lehrperson. In: Bildungsforschung. Jg. 5, Ausgabe 2. Im Internet: https://www.pedocs.de/volltexte/2014/4599/pdf/bf_2008_2_Wyss_ Reflexionsfaehigkeit.pdf. 